\title{
Aging and Neurocognitive Functioning in HIV-Infected Women: A Review of the Literature Involving the Women's Interagency HIV Study
}

\author{
David E. Vance, PhD, MGS, \\ Professor, School of Nursing, Room 2M026, 1701 University Boulevard, University of Alabama at \\ Birmingham (UAB), Birmingham, AL 35294-1210, (O) 205-934-7589, Fax: 205-996-7183 \\ Leah H. Rubin, PhD, MPH, \\ Assistant Professor, Department of Psychiatry, Room 324, MC 913, University of Illinois at \\ Chicago, Chicago, IL, 60612, (O) 312-996-6941 \\ Victor Valcour, MD, PhD, \\ Professor, UCSF School of Medicine, Department of Neurology, 3333 California Street, San \\ Francisco, CA 94104, (O) 415-476-3746 \\ Drenna Waldrop-Valverde, PhD, and \\ Associate Professor, Assistant Dean for Research, Director of Center for Neurocognitive Studies, \\ Nell Hodgson Woodruff School of Nursing, Emory University, Room 442, 1520 Clifton Road, NE, \\ Atlanta, GA 30322-4027, (O) 404-712-9487
}

\author{
Pauline M. Maki, PhD \\ Professor of Psychiatry and Psychology, Room 328, MC913, University of Illinois at Chicago, \\ Chicago, IL 60612, (O) 312-996-6941
}

David E. Vance: devance@uab.edu; Leah H. Rubin: Irubin@psych.uic.edu; Victor Valcour: Victor.Valcour@ucsf.edu; Drenna Waldrop-Valverde: drenna.waldrop-valverde@emory.edu; Pauline M. Maki: pmaki1@uic.edu

\section{Abstract}

HIV-infected women may be particularly vulnerable to certain types of neurocognitive impairments which may be exacerbated by aging and other predictors. Within the context of cognitive reserve, this article examines issues surrounding women as they age with HIV. For this, a review of 12 recent studies (2013-2016) using data from the Women's Interagency HIV Study (WIHS), the largest cohort study comparing HIV-infected and demographically-matched uninfected women, is presented that specifically examine neurocognition. In general, HIV-infected women are more vulnerable to developing neurocognitive impairments than uninfected women; other factors that may contribute to these neurocognitive impairments include recent illicit drug use, reading level (educational quality/cognitive reserve), stress, PTSD, insulin resistance, liver fibrosis, and age. Surprisingly, when examined in some analyses, age X HIV interactions were not observed to impact neurocognitive performance, findings largely consistent in the literature; however, longitudinal analyses of these data have yet to be performed which may yield future insights of how cognitive reserve may be compromised over time. Yet, with insulin resistance, 
liver fibrosis, stress, and other known predictors of poorer neurocognition also occurring more with advanced age, in time the synergistic effect of age and HIV may be more robust and observable as this population ages.

\section{Keywords}

Aging; HIV; AIDS; cognition; neurocognition; cognitive reserve women; women with HIV; HIVinfected women; MRI; memory; verbal memory; verbal learning; verbal fluency; executive function; fine motor skills; attention; WIHS; Women's Interagency HIV Study; liver fibrosis; insulin resistance; diabetes mellitus; stress; PTSD; anthropometric; neuroimaging

Since combination antiretroviral therapy (cART) blocks the replication cycle of HIV, many infected adults adherent to cART have suppressed viral loads, improved immune function, and better overall health. As a consequence, they are living longer, are aging with this disease, and are expected to have lifespans tantamount to those uninfected with HIV. ${ }^{1,2}$ By 2020 over $75 \%$ of those with HIV in the United Sates will be 50 and older. ${ }^{3}$ With $52 \%$ to $59 \%$ of HIV-infected adults already experiencing some degree of HIV-Associated Neurocognitive Disorder (HAND) ranging from Asymptomatic Neurocognitive Impairment (ANI) to HIV-Associated Dementia (HAD), ${ }^{4,5}$ those aging with this disease appear to be more vulnerable to developing HAND. In fact, one study found that older adults with HIV experience two to three times the risk of living with HAD compared to younger adults with HIV. ${ }^{6}$ Albeit, there remains debate as to whether cognitive aging in this population is accentuated (i.e., made worse than normal) or accelerated (i.e., occurs at an earlier time in the lifespan). ${ }^{7}$ Yet, this disproportionate expression in neurocognitive impairment reflects a detrimental impact in cognitive reserve in this population as they age, as well as a host of other factors that are related to cognitive reserve including sex, drug use, education, trauma, stress, comorbidities, and so forth. ${ }^{8}$

Cognitive reserve refers to how well the brain can absorb physiological insults (e.g., reduced brain perfusion, white matter hyperintensities, transient ischemic attacks, neuroinflammation, oxidative stress, drug abuse) and yet engage in optimal neurocognitive operations. Cognitive reserve normally increases during the course of one's life, especially in childhood and young adulthood when the brain is developing and maturing, in which one learns and thus builds stronger and more sophisticated connections between neurons. This neuronal growth and strengthening occurs in challenging educational attainment, occupation/employment opportunities, and social and intellectual engagement. Cognitive reserve may be supported by healthy behaviors such as physical exercise or good nutrition that simply promotes general health which benefits brain health. ${ }^{8,9}$ On the contrary, cognitive reserve may be unsupported by unhealthy behaviors such as drug use, stress, and a sedentary lifestyle. ${ }^{10-13}$ Several studies have shown that in normal older adults, ${ }^{14}$ as well as HIV-infected adults, ${ }^{10}$ active engagement facilitates increased cognitive reserve and successful cognitive aging.

Cognitive aging may be further improved by a recent clinical change of how HIV is treated clinically. Since 2013, the medical treatment for HIV has progressively emerged to current recommendations to offer cART to all HIV-infected adults, arguingthat the detrimental 
impact of viral replication on the body is immediately addressed before irreparable damage to the immune system occurs. ${ }^{15}$ This is a profound change in that everyone, regardless of disease stage, is now considered for treatment with these medications. Since HIV can cross the blood brain barrier and compromise the central nervous system, earlier initiation of cART may also produce neuroprotective effects that preserves cognitive reserve over the lifespan. ${ }^{8}$

Recent changes in timing of cART initiation could influence extenuating factors in how HIV impacts cognitive reserve and neurocognitive functioning, particularly among women. ${ }^{8}$ In some studies, ${ }^{16-19}$ these changes may be particularly influential as differential patterns and risk factors for neurocognitive functioning have been observed between HIV-infected men and women, yet such neurocognitive differences have not universally been found. ${ }^{20}$ For example, compared to HIV-infected men, HIV-infected women may be more vulnerable for developing verbal learning and verbal memory deficits. ${ }^{18}$ Also, compared to a gendermatched HIV-negative sample, women have been shown to experience poorer performance on procedural learning tasks while HIV-positive men did not. ${ }^{16}$

Furthermore, for uninfected women, menopausal stage and/or menopausal symptoms may affect neurocognitive functioning. ${ }^{21}$ For HIV-infected women, such debut of menopausal stage may occur earlier (i.e., accelerated aging) and menopausal symptoms such as psychological symptoms, sleep problems, and vasomotor symptoms may be more severe (i.e., accentuated aging) compared to uninfected women; ${ }^{22-25}$ yet, in one cross-sectional study, the severity of menopausal symptoms in each of the five categories (mood, sleep, vasomotor, somatic, vaginal) was similar in HIV-infected women and uninfected women overall and as a function of menopausal stage. In adjusted analyses, HIV-infected women remained at increased odds on persistent night sweats only. ${ }^{26}$

In addition, women are more vulnerable to neurocognitive impairments due to more exposure to trauma, physical and sexual abuse, lower education and income, barriers to health care utilization, and depression, all of which can directly or indirectly compromise physiological health as well as brain health. ${ }^{13,27}$ This point is particularly germane as women, worldwide, comprise most of those infected with HIV. ${ }^{28}$ Unfortunately, most studies that examine HIV-related neurocognitive impairment primary have focused on men or on small samples of women, which prevents the ability to reliably extrapolate what the cognitive aging profile in HIV-infected women may be.

The purpose of this article is to provide an updated review current HIV-related neurocognitive literature (published from 2013 to 2016) that reflects recent medical standards of HIV treatment as it pertains particularly to women with HIV, especially as they age. To accomplish this goal, the source material for extrapolating the neurocognitive profile of HIV-infected women as they age comes from the Women's Interagency HIV Study (WIHS), which is the largest cohort study of HIV-infected women. These most recent neurocognitive and brain imaging studies from this large, comprehensive dataset are summarized (Table 1) and then synthesized as they relate to cognitive reserve and cognitive aging.

Curr HIV/AIDS Rep. Author manuscript; available in PMC 2017 December 01. 


\section{Overview of the WIHS's Neurocognitive Protocol}

WIHS is the largest cohort study following HIV-infected women which also has a demographically-matched sample of uninfected women. These women are followed longitudinally and are administered a variety of psychosocial and physiological measures (e.g., CD4 cell count, viral load, liver function tests) every 6 months, with four visits occurring over a 2-year period. WIHS has been on-going since October 1994 and has a rich database supported by a coordinating center (Johns Hopkins University) with several current/past sites (Brooklyn, NY; Bronx/Manhattan, NY; Chicago, IL; Los Angeles, CA; San Francisco/Bay Area, CA; Washington, DC) and newly added southern sites (Atlanta, GA; Chapel Hill, NC; Birmingham, AL; Jackson, MS; Miami, FL). ${ }^{29,30}$

All of the current WIHS neurocognitive protocols published in 2013-2016 share the following strengths and limitations. First, all are cross-sectional and use the data from the original sites; although WIHS has incorporated an additional follow-up wave of neurocognitive data, these longitudinal data are only recently becoming available for analysis. Second, with such a large generalizable urban sample, all of these analyses are statistically able to incorporate a rich array of covariates which are available to control for potential confounds. These covariates vary slightly between the various articles and even within the individual articles themselves depending on the aims within each secondary analysis. In some studies, some of these variables are controled, while in others, normedbased regression scores are used to handle these covariates. Although not comprehensive, the typical covariates include: age; study site; annual household income; Center for Epidemiological Studies Depression Scale (CES-D cutoff $\geq 16$ ); hepatitis C status; antidepressant use; smoking status (recent, former, never); marijuana, cocaine, and/or heroin use (recent, former, never); recent heavy alcohol use; and, prior exposure to neurocognitive tests. Third, although age was not a primary focus in most of these studies, the sample itself represents mostly middle-aged women in their 40's with a large age range across the lifespan. Fourth, all of the sites conformed to a three-step certification process to train testers to administer the neurocognitive battery without "drift" in the protocol, thus ensuring quality data collection. Fourth, the neurocognitive test battery was selected by a panel of experts that would allow a HAND diagnosis to be calculated using the Antinori et al. research nosology ${ }^{31}$ as well as comparisons to be made with the Multicenter AIDS Cohort Study (MACS) which focuses specifically on HIV-infected men; ${ }^{30,31}$ however, at the moment, none of the WIHS studies have incorporated the HAND diagnosis yet.

From October 2004 to September 2006 as part of the WIHS core assessment (visits 21-24), participants were administered the Trails Making Test and the Symbol Digit Modalities Test. Between October 2006 and September 2008, the Comalli-Kaplan Stroop Test was also administered (visits 25-28). The Hopkins Verbal Learning Test was administered between April 2007 - 2008. Then in 2009, the full standardized, neurocognitive battery was incorporated in the assessment visits which measure seven cognitive domains: 1) Fine Motor Skills (Grooved Pegboard Test); 2) Psychomotor Speed (Symbol Digit Modalities Test); 3) Attention (Stroop Test, trials $1 \&$ 2; Trails Making Test Part A; Letter-Number Sequence (LNS) Test, control/attention condition); 4) Executive Function composed of working/verbal memory, behavioral inhibition, and mental flexibility (Stroop Test, Trial 3 of the color-word 
interference condition; Trails Making Test Part B; LNS Test, working memory condition); 5) Verbal Learning (Hopkins Verbal Learning Test - Revised (HVLT-R), single trial learning and total words recalled across the three trials); 6) Verbal Memory (HVLT-R delayed recall $\&$ percent retention); and 7) Verbal Fluency (Letter Fluency Task \& Category Fluency Task). ${ }^{13,27,30}$ Finally, these neurocognitive scores are examined either raw (unadjusted) or adjusted (e.g., multivariable models, ${ }^{32-34}$ propensity score methods ${ }^{27}$ ). In addition, in some of the studies, the cognitive domains were averaged by deriving $t$-scores within the individual tests within the cognitive domain; however, this varies by analysis within each article. Since these $t$-scores are derived internally, the WIHS avoids bias associated with external norming, a factor that is particularly important for this cohort that is rich with diversity in race and lifestyle behaviors and is made up entirely of women.

The sample characteristics for these cross-sectional analyses are for data collected 2009 to 2011. Of the 1,908 women in WIHS, $84 \%$ completed the neurocognitive battery. ${ }^{30}$ Approximately $60 \%$ of the women are African American, $30 \%$ have significant depressive symptomatology (CES-D cutoff $\geq 16$ ), and the average educational level is around 12 years (high school graduate).$^{30}$ The average age of the sample was 46.15 years (range: 25 to 87 ), and $45 \%$ has an annual household income $\leq \$ 12,000 /$ year. With missing data and using listwise deletion, for most of the WIHS neurocognitive articles presented here, approximately 1,000 HIV-infected and 500 uninfected women are available for most analyses. Of the HIV-infected women, approximately $37 \%$ had suboptimal cART adherence, 47\% had detectable HIV RNA in their plasma, and $87 \%$ had a CD4+ T-cell count $>200$ cells/mcL, which is above the AIDS criteria. ${ }^{35}$ The frequency of viral suppression falls between that reported by the CHARTER (CNS HIV Antiretroviral Therapy Effects Research) and MACS cohorts and requires separate sensitivity analyses to understand the impact of HIV on cognition among suppressed participants, a population increasingly pertinent to clinical care.

\section{WHIS Neurocognitive and Brain Imaging Studies}

The WIHS neurocognitive articles published in the past three years focus on predictors on neurocognitive functioning and/or brain imaging differences between and among HIVinfected and uninfected women. Study details for these have been briefly summarized above and more specifically in Table 1 . Few of these studies specifically examined age as a predictor or interaction with HIV; yet, given that the average sample age is in the mid 40's with a wide range in age, the results provide a context in which to consider cognitive aging in this clinical population.

\section{Neurocognitive Comparisons of Women with and without HIV}

The focus on predictors of neurocognition in the WIHS range from perceived stress to liver fibrosis. Instead of presenting the articles in chronological order in which they were published, they have been grouped by category: Psychosocial Predictors, and Biological Predictors.

Psychosocial Predictors-In one of WIHS's overarching analyses of cognition, Maki and colleagues ${ }^{30}$ examined the relationship and magnitude between neurocognition and HIV 
status across several predictors. In general, compared to uninfected women, HIV-infected women as a group performed worse on measures of attention, psychomotor speed, verbal learning, delayed (memory) recall, and recognition. Overall, the greatest HIV impairment was in verbal memory (Cohen $\delta=-.20$ ). Also, it was found that the effect size for HIV on neurocognition was very small, ranging from .05 to $.09 \mathrm{SD}$ units. In comparison, the effect size on neurocognition was larger for age, race/ethnicity, annual household income, years of education, reading level (e.g., Wide Range Achievement Test-3, an indicator of education quality), and in some cases, depressive symtomatology. In fact, reading level yielded the strongest effect sizes for predicting neurocognition in attention as well as verbal learning and verbal memory, ranging from 0.15 to 0.35 SD units. Furthermore, HIV status and reading level produced a significant interaction on measures of executive and psychomotor function; this finding suggests infected women with poorer reading level are more susceptible to developing neurocognitive impairments.

Stress is a unique psychosocial predictor of neurocognition in which HIV-infected women may be particularly vulnerable. In this next WIHS article, Rubin and colleagues ${ }^{13}$ examined the association between stress, as measured by the Perceived Stress Scale. As expected, HIV serostatus was associated with poorer performance on verbal learning, verbal memory, and attention; however, for both HIV-infected and uninfected women, higher stress was associated with poorer verbal learning, verbal memory, attention, speed of information processing, and executive functioning. Notably, there was an interaction between HIV and stress indicating that in the context of HIV, women experiencing more stress exhibited poorer verbal memory compared to women experiencing less stress. Furthermore, this pattern was exacerbated among HIV+ women with viral loads $>10,000$ copies $/ \mathrm{ml}$. Similarly, in HIV-infected men, Pukay-Martin and colleagues ${ }^{36}$ found that acute stressful life events was also associated with worse executive functioning, attention, and processing speed, but not memory.

In a related analysis, Rubin and colleagues ${ }^{27}$ investigated the role of post-traumatic stress disorder (PTSD) on neurocognitive functioning. This examination is of particular importance because compared to uninfected women in the United States: 1) childhood sexual abuse in HIV-infected women is twice as great, ${ }^{37,38}$ and 2) HIV-infected women are three times more likely to experience PSTD. ${ }^{39-41}$ In this analysis, HIV status had little interaction with PTSD status; in fact, women with PTSD performed worse on measures of verbal memory, verbal learning, and psychomotor speed. However, the association of PTSD on neurocognitive functioning varied depending on the exposure to sexual abuse and/or violence. Specifically, the exposure to violence or sexual abuse was associated with a greater number of cognitive domains and an overall worse neurocognitive profile. The only significant interaction between HIV and PTSD revealed that uninfected women with PTSD performed worse on a measure of fine motor skills compared to HIV-infected women with PTSD. This study is in contrast to a study examining the relationship between HIV-infected men with PTSD, which found that PTSD was only associated with memory; ${ }^{42}$ however, both studies used a different neurocognitive battery and a different measure of PTSD.

Another psychosocial factor that can impact neurocognitive functioning is drug use. Meyer and colleagues ${ }^{12}$ examined the role in which HIV interacts with drug use to impact 
neurocognition. Drug use was categorized in three decreasing frequencies: recent drug use, former drug use, and non-use. These researchers found that recent drug use and HIV infection each contributed to poorer verbal learning and verbal memory; however, the interactions between HIV serostatus and recent drug use, compared to non-use, was associated with poorer verbal learning and memory; specifically, the combination of HIV and recent drug use exacerbate neurocognitive impairment in these domains. Yet, there was no HIV X recent drug use on measures of executive functioning and information speed of processing. Given that women have a greater tendency to use cocaine currently or over the lifespan than men, ${ }^{43}$ and that cocaine use may accelerate HIV progression, ${ }^{44}$ these finds are particularly compelling as the interaction between HIV progression and recent drug use may compromise the neurocircuitry that supports these neurocognitive abilities. This study used WIHS visit 25 data that only included the Stroop and HVLT-R; thus, more work is needed to see if this replicates and whether the effects are specific to learning and memory since this was not examined.

Biological Predictors-Several biological predictors have been examined in WIHS including menopause staging, anthropological measures, insulin resistance, and liver fibrosis. Unique to women, menopausal stage/symptoms may debut earlier/more severely in HIV-infected women. ${ }^{22-25}$ Studies in uninfected women suggest that menopausal stage, and/or menopausal symptoms such as vasomotor, sleep, and mood symptomatology, can affect neurocognitive functioning. ${ }^{21}$ In addition, studies involving animals and humans show that estrogen influences the prefrontal cortex and hippocampus, which are brain regions important for verbal learning and memory. ${ }^{45,46}$ To examine this potential relationship, Rubin and colleagues ${ }^{47}$ examined the staging of menopause (early perimenopause, late perimenopause, \& postmenopause) and accompanying menopausal symptoms on neurocognition. Overall, HIV infection, but not menopausal stage, was related to poorer attention, memory, and executive functioning. However, menopausal symptoms were inversely associated with neurocognitive functioning. Whereas anxiety symptoms were also associated with poorer verbal learning and memory, depressive symptoms were related to these two cognitive abilities as well as poorer attention and executive functioning. Additionally, greater vasomotor symptoms were related to poorer attention. Furthermore, the association between anxiety symptoms and verbal learning was moderated by HIV status such that elevated anxiety was associated with worse verbal learning in HIV-infected women only. It was surprising that menopausal staging was not observed to differentially affect cognition in these women; however, other studies in uninfected, more educated Caucasian women (e.g., SWAN) detected such changes longitudinally. ${ }^{21}$ Likewise, this sample of HIVinfected women was comprised of more African Americans, were less educated and less economically stable, engaged in more drug use, and experienced a higher prevalence of depression than studies with uninfected women showing such menopausal effects of cognition. $^{21}$

In another WIHS study, Gustafson and colleagues ${ }^{48}$ examined the association of anthropometric measures (i.e., waist-to-hip ratio, waist circumference, and body mass index) on neurocognition in middle-aged women; this study used WIHS visit $21-24$ data that only included the Trails Making Test and the Stroop Test, so the full comprehensive battery was 
not examined. These researchers found that among HIV-infected women, using BMI between $20-24.9 \mathrm{~kg} / \mathrm{m}^{2}$ as a reference group, a BMI of $<18.5 \mathrm{~kg} / \mathrm{m}^{2}$ was associated with poorer executive functioning and speed of information processing. Meanwhile, among HIVinfected women, a BMI $\geq 30.0 \mathrm{~kg} / \mathrm{m}^{2}$ was associated with better executive functioning. Yet, for HIV-infected women, a BMI $230.0 \mathrm{~kg} / \mathrm{m}^{2}$ was associated with poorer speed of information processing. Furthermore, a waist circumference greater than $88 \mathrm{~cm}$ was associated with better executive functioning while those with a waist circumference in the middle category $(80-87.9 \mathrm{~cm})$ performed best on attention. Similarly, a greater waist-to-hip ratio was associated with speed of information processing. Although the results are somewhat mixed, in general higher anthropometric values are associated with better neurocognition in HIV-infected women, which is rather contradictory compared to studies examining uninfected women, a finding that may, in part, be due to body habitus changes associated with antiretroviral exposure that could complicate the link of BMI to cardiovascular and brain health outcomes. ${ }^{49}$ This may also reflect a restoration of health linked to higher BMIs, a testament to the efficacy of these HIV medications that also may be neuroprotective as suggested in the CHARTER study. ${ }^{50,51}$ This point is particularly relevant as over $80 \%$ of this sample of women with HIV were taking cART.

Although cART has been shown to improve neurocognition in HIV-infected adults, ${ }^{50}$ these medications may contribute to metabolic disorders, such as diabetes mellitus and insulin resistance, which diminishes neurocognitive function. ${ }^{51}$ In a sample of $658 \mathrm{HIV}$-infected and uninfected women without diabetes, Valcour and colleagues ${ }^{32}$ examined the relationship between insulin resistance, measured by the homeostasis model assessment (HOMA) and neurocognition. Elevated HOMA (greater insulin resistance) predicted poorer attention and verbal fluency. Furthermore, an HIV X HOMA interaction was observed; specifically, in HIV-infected women, elevated HOMA predicted poorer attention. In a separate analysis including WIHS participants with diabetes mellitus, this metabolic disorder predicted poorer function in attention and psychomotor speed. These findings confirm previous research on diabetes mellitus and insulin resistance on neurocognition. ${ }^{34}$

Since HIV also impairs gut epithelium, it has been suggested that Minimal Hepatic Encephalopathy may also contribute to poorer neurocognition, particularly among participants with both HIV and HCV. To examine this, Valcour and colleagues ${ }^{33}$ examined the associated between cognitive correlates and liver fibrosis, using the aspartate aminotransferase to platelet ratio index (APRI) and Fibroscan, in HIV-infected and uninfected women with and without Hepatitis C. Using APRI, moderate/severe liver fibrosis was associated with poorer fine motor skills, psychomotor speed, verbal learning, verbal memory, verbal fluency, and executive functioning. Using FibroScan, severe liver fibrosis was associated with poorer verbal fluency, attention, and executive functioning. This study showed that independent of HIV and hepatitis $\mathrm{C}$, liver fibrosis has a unique contribution to neurocognitive impairment; however, authors caution that a common pathway to both cognitive outcomes and liver fibrosis is chronic inflammation. Thus, both cognitive outcomes and fibrosis may be a result of inflammation rather than causative of each other. 


\section{Brain Imaging Studies}

Four brain imaging studies have been conducted in WIHS within the past 3 years. Given the nature and cost of neuroimaging studies, the sample sizes are expectedly small (Table 1).

In this first study, Liu and colleagues ${ }^{52}$ used advanced fMRI techniques to examine if neural specificity, an index of neuronal dysfunction, is observed in HIV-infected women before structural changes in the brain or neurocognitive impairments are detectable. In examining the fusiform face area in the temporal lobe, 15 HIV-infected and 13 uninfected women were administered a face discrimination test outside the scanner as well as an in-scanner face discrimination task. Both groups of women performed comparably on the behavioral neurocognitive face discrimination test outside the scanner. Neural specificity was defined as "an earlier recovery from adaptation" and a narrow versus a broadened neural activation in the fusiform face area during the in-scanner face discrimination task. Neural specificity was decreased in HIV-infected women. This decreased neural specificity suggests that before behavioral neurocognitive changes or structural changes in the brain emerge, neuronal dysfunction is occurring in HIV-infected women.

In this second study, Meyer and colleagues ${ }^{11}$ examined the effect of crack cocaine use on verbal memory and prefrontal cortical dysfunction in $30 \mathrm{HIV}$-infected women. A memory task-based fMRI paradigm was used to examine brain activation/dysfunction while participants engaged an in-scanner verbal memory task that paralleled the HVLT-R so that semantic clustering could be studied. Those currently using crack cocaine performed poorer on verbal memory, specifically semantic clustering. During the encoding task, left anterior cingulate cortex activation was higher in those who never used crack cocaine compared to those who were current and former users. During the recognition task, bilaterial dorsolateral prefrontal cortex and left dorsal medial prefrontal cortex activation was higher in those who never used crack cocaine compared to those who were current and former users.

Furthermore, poorer performance on the recognition task correlated with lower activation in the left dorsolateral prefrontal cortex. Thus, dysfunction in the prefrontal cortex and the anterior cingulate cortex may account for poorer verbal memory and verbal learning associated with crack cocaine use.

In this next study, Rubin and colleagues ${ }^{53}$ examined the effect of stress on verbal memory and brain volume in 38 HIV-infected women. The Perceived Stress Scale (PSS-10) was used to categorize participants between lower stress (lowest two tertiles $<18$ ) and higher stress levels (highest tertile 218 ) and structural MRI was used to examine brain volume. HIVinfected women with higher stress levels performed worse on measures of verbal memory and strategic retrieval and showed smaller volumes in the prefrontal cortex, and specifically in the superior, medial, and inferior frontal gyrus and the parahippocampal gyrus (bilaterally). In addition, poorer verbal learning and memory were associated with smaller brain volumes in the superior, medial, and inferior frontal gyrus (right hemisphere).

Finally, in a similar study, Rubin and colleagues ${ }^{54}$ examined the effect stress had on verbal memory and prefrontal cortical dysfunction in $36 \mathrm{HIV}$-infected women. As in the study before, the Perceived Stress Scale (PSS-10) was used to categorize participants between lower stress (lowest two tertiles < 18) and higher stress levels (highest tertile $\geq 18$ ). A 
memory task-based fMRI paradigm was used to examine brain activation/dysfunction while participants. HIV-infected women with higher stress levels performed worse on measures of verbal memory including strategic retrieval. During the verbal recognition task, women with higher stress levels displayed greater deactivation in the posterior cingulate cortex and the medial prefrontal lobe. Similar findings were observed when examining PTSD symptoms but not depressive symptoms.

\section{Discussion}

Collectively, these recent WIHS neurocognitive studies tell a unique story about HIVinfected women. First, compared to uninfected women, HIV-infected women in general perform worse neurocognitively, especially on measures of verbal learning and verbal memory; and this finding is consistent across studies even when either controlling for a number covariates or examining for particular predictors. In contrast, HIV-infected men are observed to experience worse neurocognitive functioning in executive functioning and learning. 55,56

Second, the effect of HIV on neurocognitive impairment is small compared to other predictors such as age, income, race, and more importantly, reading level, which is a proxy of cognitive reserve. ${ }^{30}$ The diminished impact of HIV compared to demographically matched populations is consistent with published work. Although menopausal stage did not exert any detrimental neurocognitive effects, clearly drug use, stress-related factors, insulin resistance and diabetes mellitus, and liver fibrosis (likely a marker of general inflammation) does and may interact with HIV to accentuate or accelerate cognitive aging.

In fact, as this population ages, the prevalence and severity of some conditions will rise; for example, the prevalence of diabetes mellitus occurs in $28.8 \%$ of HIV-infected adults 60 and older, ${ }^{57}$ which may further compromise cognitive reserve. ${ }^{58}$ In addition, studies in uninfected adults show that with advancing age, women perceive more stress than men. ${ }^{59}$ Such stress may lead to metabolic disorders such as insulin resistance and diabetes. ${ }^{60,61}$ Furthermore, it is well documented that HIV-infected women experience a high number of stressors. Among HIV-infected women in the WIHS, 31\% reported childhood sexual abuse, $66 \%$ reported domestic violence within their lifetime, and $21 \%$ reported domestic violence within the past 3 months. ${ }^{62}$ With stress exerting a detrimental effect on the aging brain and neurocognition, ${ }^{63}$ accompanied with a sharp decrease in gonadal hormones in women compared to men, ${ }^{64}$ this may further compromise cognitive reserve, especially for HIVinfected women for which these conditions are common.

Third, as seen in the Maki and colleagues study, ${ }^{30} \mathrm{HIV}$-infected women with lower cognitive reserve, as measured by lower reading level, were more vulnerable to having neurocognitive impairments. Surprisingly, although age was a predictor of poorer neurocognition in general, an age X HIV interaction was not observed; perhaps this was because of the age of the samples were mainly in the middle 40's before substantial age-related impairments have the opportunity to be expressed with HIV. Models used in the WIHS analysis did not separately examine age X HIV effects among women who have met AIDS diagnostic criteria, a group most vulnerable to these interactions effects in past published work. ${ }^{65,66}$ Age X HIV 
interactions were not the primary focus of these WIHS neurocognitive studies, but in general, the larger HIV literature does not show strong interaction effects, although there are strong trends in showing more neurocognitive impairment in older adults with HIV. ${ }^{67,68}$

As more longitudinal data become available in the WIHS, it may be that such age X HIV interactions or more complex interactions between age X HIV X reading level (i.e., cognitive reserve) will be observed, with probable neurocognitive impairments being observed in verbal learning and verbal memory in HIV-infected women who already have a lower cognitive reserve. The potential interactions may be especially pronounced if these women experience high levels of stress, insulin resistance, and so forth. In addition, other analysis may also examine the impact of poor antiretroviral adherence and lack of sustained viral suppression on neurocognition. Clearly, there are a number of potential factors that may interact with each other to better predict neurocognition over time.

Fourth, the few recent WHIS neuroimaging studies show, compared to uninfected women, reduce neural specificity as well as impairments and/or volume loss in the prefrontal cortex, anterior cingulate, the parahippocampal gyrus, and frontal gyrus were observed in the HIVinfected women. These regional impairments were also associated with greater levels of stress and cocaine use, and correspond to poorer performance with verbal learning and memory. ${ }^{11,53}$ It has been observed in normal aging that such age-related impairments in these regions are also observed ${ }^{69}$ which may predispose HIV-infected women to additional declines in cognitive reserve. The imaging studies completed in the WIHS also highlights substantial gaps in the examination of brain structure and function among HIV-infected women when comparing the number of studies completed compared to the number published among HIV-infected men.

\section{Conclusion}

Our review of the most recent WIHS neurocognitive studies highlights the most comprehensive summary on the literature documenting the impact of HIV and related neurocognitive predictors on the largest cohort of HIV-infected and uninfected women. So far, HIV has shown a consistent pattern in that HIV-infected women are more vulnerable to impairments in verbal memory and verbal learning in particular; these impairments may be exacerbated by drug use, stress, PTSD, insulin resistance, and liver fibrosis. As some of these neurocognitive predictors may become more severe or more prevalent with advancing age, this suggests that such age-related factors may exacerbate such neurocognitive impairment, especially for those with poorer cognitive reserve (i.e., reading level). Yet, these age-related factors when statistically controlled may obscure the independent effect that age itself exerts on neurocognition; it may be for this reason that age X HIV interactions are inconsistency observed in HIV-infected women as well as men.

Future studies in WIHS will use the HAND diagnosis to examine predictors of change over time. Antinori and colleagues ${ }^{31}$ observed that over a 1-year period, classification of HAND can vary as much as $20 \%$. With our neurocognitive battery being administered every 2 years, it will be of particular interest whether this same phenomenon is observed. And more 
importantly, discovering what factors predict those who improve or worsen will be vital for developing effective interventions to preserve cognitive reserve as HIV-infected women age.

\section{Supplementary Material}

Refer to Web version on PubMed Central for supplementary material.

\section{References}

Papers of special interest, published recently, have been denoted as:

** Of major importance.

1. Rodger AJ, Lodwick R, Schechter M, et al. Mortality in well controlled HIV in the continuous antiretroviral therapy arms of the SMART and ESPRIT trials compared with the general population. AIDS. 2013; 27(6):973-979. [PubMed: 23698063]

2. Lewden C, Bouteloup V, et al. Collaboration of Observational HIVEREiE. All-cause mortality in treated HIV-infected adults with CD4 $>/=500 / \mathrm{mm} 3$ compared with the general population: evidence from a large European observational cohort collaboration. Int J Epidemiol. 2012; 41(2):433-445. [PubMed: 22493325]

3. Deeks SG, Lewin SR, Havlir DV. The end of AIDS: HIV infection as a chronic disease. Lancet. 2013; 382(9903):1525-1533. [PubMed: 24152939]

4. Heaton RK, Clifford DB, Franklin DR Jr, et al. HIV-associated neurocognitive disorders persist in the era of potent antiretroviral therapy: CHARTER Study. Neurology. 2010; 75(23):2087-2096. [PubMed: 21135382]

5. Bonnet F, Amieva H, Marquant F, et al. Cognitive disorders in HIV-infected patients: are they HIVrelated? AIDS. 2013; 27(3):391-400. [PubMed: 23079813]

6. Valcour V, Shikuma C, Shiramizu B, et al. Higher frequency of dementia in older HIV-1 individuals: the Hawaii Aging with HIV-1 Cohort. Neurology. 2004; 63(5):822-827. [PubMed: 15365130]

7. Pathai S, Bajillan H, Landay AL, High KP. Is HIV a model of accelerated or accentuated aging? J Gerontol A Biol Sci Med Sci. 2014; 69(7):833-842. [PubMed: 24158766]

8. Cody SL, Vance DE. The neurobiology of HIV and its impact on cognitive reserve: A review of cognitive interventions for an aging population. Neurobiol Dis. 2016

9. Vance DE, Cody SL, Yoo-Jeong M, Jones GL, Nicholson WC. The Role of Employment on Neurocognitive Reserve in Adults With HIV: A Review of the Literature. J Assoc Nurses AIDS Care. 2015; 26(4):316-329. [PubMed: 26066688]

10. Fazeli PL, Woods SP, Heaton RK, et al. An active lifestyle is associated with better neurocognitive functioning in adults living with HIV infection. J Neurovirol. 2014; 20(3):233-242. [PubMed: 24554483]

11. Meyer VJ, Little DM, Fitzgerald DA, et al. Crack cocaine use impairs anterior cingulate and prefrontal cortex function in women with HIV infection. J Neurovirol. 2014; 20(4):352-361. [PubMed: 24760360]

12. Meyer VJ, Rubin LH, Martin E, et al. HIV and recent illicit drug use interact to affect verbal memory in women. J Acquir Immune Defic Syndr. 2013; 63(1):67-76. [PubMed: 23392462]

13. Rubin LH, Cook JA, Weber KM, et al. The association of perceived stress and verbal memory is greater in HIV-infected versus HIV-uninfected women. J Neurovirol. 2015; 21(4):422-432. [PubMed: 25791344]

14. Park DC, Lodi-Smith J, Drew L, et al. The impact of sustained engagement on cognitive function in older adults: the Synapse Project. Psychol Sci. 2014; 25(1):103-112. [PubMed: 24214244]

15. De Cock KM, El-Sadr WM. When to start ART in Africa--an urgent research priority. N Engl J Med. 2013; 368(10):886-889. [PubMed: 23425131] 
16. Martin E, Gonzalez R, Vassileva J, Maki P. HIV+ men and women show different performance patterns on procedural learning tasks. J Clin Exp Neuropsychol. 2011; 33(1):112-120. [PubMed: 20694870]

17. Royal W 3rd, Cherner M, Burdo TH, et al. Associations between Cognition, Gender and Monocyte Activation among HIV Infected Individuals in Nigeria. PLoS One. 2016; 11(2):e0147182. [PubMed: 26829391]

18. Maki, PM.; Rubin, LH.; Springer, G., et al. Male/female differences in cognitive function in HIV+ individuals. Conference on Retroviruses and Opportunistic Infections (CROI); February 2016; Boston, MA.

19. Martin E, Gonzalez R, Vassileva J, Maki PM, Bechara A, Brand M. Sex and HIV serostatus differences in decision making under risk among substance-dependent individuals. J Clin Exp Neuropsychol. 2016; 38(4):404-415. [PubMed: 26882176]

20. Behrman-Lay AM, Paul RH, Heaps-Woodruff J, Baker LM, Usher C, Ances BM. Human immunodeficiency virus has similar effects on brain volumetrics and cognition in males and females. J Neurovirol. 2016; 22(1):93-103. [PubMed: 26306688]

21. Greendale GA, Wight RG, Huang MH, et al. Menopause-associated symptoms and cognitive performance: results from the study of women's health across the nation. Am J Epidemiol. 2010; 171(11):1214-1224. [PubMed: 20442205]

22. Ferreira CE, Pinto-Neto AM, Conde DM, Costa-Paiva L, Morais SS, Magalhaes J. Menopause symptoms in women infected with HIV: prevalence and associated factors. Gynecol Endocrinol. 2007; 23(4):198-205. [PubMed: 17505939]

23. Boonyanurak P, Bunupuradah T, Wilawan K, et al. Age at menopause and menopause-related symptoms in human immunodeficiency virus-infected Thai women. Menopause. 2012; 19(7):820824. [PubMed: 22549170]

24. Schoenbaum EE, Hartel D, Lo Y, et al. HIV infection, drug use, and onset of natural menopause. Clin Infect Dis. 2005; 41(10):1517-1524. [PubMed: 16231267]

25. Kanapathipillai R, Hickey M, Giles M. Human immunodeficiency virus and menopause. Menopause. 2013; 20(9):983-990. [PubMed: 23531684]

26. Rubin, LH.; Sundermann, E.; Golub, ET., et al. The influence of serostatus and menopausal stage on menopausal symptoms in the Women's Interagency HIV Study: Are HIV+ women at increased risk for menopausal symptoms?. North America Menopause Society 20th Annual Meeting; September, 2009; San Diego, CA.

27. Rubin LH, Pyra M, Cook JA, et al. Post-traumatic stress is associated with verbal learning, memory, and psychomotor speed in HIV-infected and HIV-uninfected women. J Neurovirol. 2016; 22(2):159-169. [PubMed: 26404435]

28. WHO. Women and health: Today's evidence tomorrow's agenda. Geneva, Switzerland: WHO Press; 2009.

29. Bacon MC, von Wyl V, Alden C, et al. The Women's Interagency HIV Study: an observational cohort brings clinical sciences to the bench. Clin Diagn Lab Immunol. 2005; 12(9):1013-1019. [PubMed: 16148165]

30. Maki PM, Rubin LH, Valcour V, et al. Cognitive function in women with HIV: findings from the Women's Interagency HIV Study. Neurology. 2015; 84(3):231-240. [PubMed: 25540304]

31. Antinori A, Arendt G, Becker JT, et al. Updated research nosology for HIV-associated neurocognitive disorders. Neurology. 2007; 69(18):1789-1799. [PubMed: 17914061]

32. Valcour V, Rubin LH, Tien P, et al. Human immunodeficiency virus (HIV) modulates the associations between insulin resistance and cognition in the current combination antiretroviral therapy (cART) era: a study of the Women's Interagency HIV Study (WIHS). J Neurovirol. 2015; 21(4):415-421. [PubMed: 25740539]

33. Valcour VG, Rubin LH, Obasi MU, et al. Liver Fibrosis Linked to Cognitive Performance in HIV and Hepatitis C. J Acquir Immune Defic Syndr. 2016

34. Valcour VG, Sacktor NC, Paul RH, et al. Insulin resistance is associated with cognition among HIV-1-infected patients: the Hawaii Aging With HIV cohort. J Acquir Immune Defic Syndr. 2006; 43(4):405-410. [PubMed: 17099311] 
35. Cysique LA, Becker JT. Lessons to be learned from the largest study of cognition in American women with HIV disease. Neurology. 2015; 84(3):220-221. [PubMed: 25540319]

36. Pukay-Martin ND, Cristiani SA, Saveanu R, Bornstein RA. The relationship between stressful life events and cognitive function in HIV-infected men. J Neuropsychiatry Clin Neurosci. 2003; 15(4): 436-441. [PubMed: 14627770]

37. Cougle JR, Timpano KR, Sachs-Ericsson N, Keough ME, Riccardi CJ. Examining the unique relationships between anxiety disorders and childhood physical and sexual abuse in the National Comorbidity Survey-Replication. Psychiatry Res. 2010; 177(1-2):150-155. [PubMed: 20381878]

38. Machtinger EL, Wilson TC, Haberer JE, Weiss DS. Psychological trauma and PTSD in HIVpositive women: a meta-analysis. AIDS Behav. 2012; 16(8):2091-2100. [PubMed: 22249954]

39. Kessler RC, Petukhova M, Sampson NA, Zaslavsky AM, Wittchen HU. Twelve-month and lifetime prevalence and lifetime morbid risk of anxiety and mood disorders in the United States. Int $\mathbf{J}$ Methods Psychiatr Res. 2012; 21(3):169-184. [PubMed: 22865617]

40. Kessler RC, Sonnega A, Bromet E, Hughes M, Nelson CB. Posttraumatic stress disorder in the National Comorbidity Survey. Arch Gen Psychiatry. 1995; 52(12):1048-1060. [PubMed: 7492257]

41. Breslau N, Kessler RC, Chilcoat HD, Schultz LR, Davis GC, Andreski P. Trauma and posttraumatic stress disorder in the community: the 1996 Detroit Area Survey of Trauma. Arch Gen Psychiatry. 1998; 55(7):626-632. [PubMed: 9672053]

42. Moradi AR, Miraghaei MA, Parhon H, Jabbari H, Jobson L. Posttraumatic stress disorder, depression, executive functioning, and autobiographical remembering in individuals with HIV and in carers of those with HIV in Iran. AIDS Care. 2013; 25(3):281-288. [PubMed: 22775122]

43. Lejuez CW, Bornovalova MA, Reynolds EK, Daughters SB, Curtin JJ. Risk factors in the relationship between gender and crack/cocaine. Exp Clin Psychopharmacol. 2007; 15(2):165-175. [PubMed: 17469940]

44. Baum MK, Rafie C, Lai S, Sales S, Page B, Campa A. Crack-cocaine use accelerates HIV disease progression in a cohort of HIV-positive drug users. J Acquir Immune Defic Syndr. 2009; 50(1):9399. [PubMed: 19295339]

45. Maki PM, Resnick SM. Effects of estrogen on patterns of brain activity at rest and during cognitive activity: a review of neuroimaging studies. Neuroimage. 2001; 14(4):789-801. [PubMed: 11554798]

46. Resnick SM, Maki PM. Effects of hormone replacement therapy on cognitive and brain aging. Ann N Y Acad Sci. 2001; 949:203-214. [PubMed: 11795355]

47. Rubin LH, Sundermann EE, Cook JA, et al. Investigation of menopausal stage and symptoms on cognition in human immunodeficiency virus-infected women. Menopause. 2014; 21(9):997-1006. [PubMed: 24496085]

48. Gustafson DR, Mielke MM, Tien PC, et al. Anthropometric measures and cognition in middleaged HIV-infected and uninfected women. The Women's Interagency HIV Study. J Neurovirol. 2013; 19(6):574-585. [PubMed: 24338243]

49. Fitzpatrick AL, Kuller LH, Lopez OL, et al. Midlife and late-life obesity and the risk of dementia: cardiovascular health study. Arch Neurol. 2009; 66(3):336-342. [PubMed: 19273752]

50. Al-Khindi T, Zakzanis KK, van Gorp WG. Does antiretroviral therapy improve HIV-associated cognitive impairment? A quantitative review of the literature. J Int Neuropsychol Soc. 2011; 17(6): 956-969. [PubMed: 21813033]

51. McCutchan JA, Marquie-Beck JA, Fitzsimons CA, et al. Role of obesity, metabolic variables, and diabetes in HIV-associated neurocognitive disorder. Neurology. 2012; 78(7):485-492. [PubMed: 22330412]

52. Liu C, Wang C, Leclair M, Young M, Jiang X. Reduced neural specificity in middle-aged HIV+ women in the absence of behavioral deficits. Neuroimage Clin. 2015; 8:667-675. [PubMed: 26288750]

53. Rubin LH, Meyer VJ, RJC, et al. Prefrontal cortical volume loss is associated with stress-related deficits in verbal learning and memory in HIV-infected women. Neurobiol Dis. 2015

54. Rubin LH, Wu M, Sundermann EE, et al. Elevated stress is associated with prefrontal cortex dysfunction during a verbal memory task in women with HIV. J Neurovirol. 2016

Curr HIV/AIDS Rep. Author manuscript; available in PMC 2017 December 01. 
55. Heaton RK, Franklin DR, Ellis RJ, et al. HIV-associated neurocognitive disorders before and during the era of combination antiretroviral therapy: differences in rates, nature, and predictors. $\mathrm{J}$ Neurovirol. 2011; 17(1):3-16. [PubMed: 21174240]

56. Woods SP, Scott JC, Dawson MS, et al. Construct validity of Hopkins Verbal Learning TestRevised component process measures in an HIV-1 sample. Arch Clin Neuropsychol. 2005; 20(8): 1061-1071. [PubMed: 16198529]

57. Vance DE, Mugavero M, Willig J, Raper JL, Saag MS. Aging with HIV: a cross-sectional study of comorbidity prevalence and clinical characteristics across decades of life. J Assoc Nurses AIDS Care. 2011; 22(1):17-25. [PubMed: 20471864]

58. Vance DE, Fazeli PL, Dodson JE, Ackerman M, Talley M, Appel SJ. The synergistic effects of HIV, diabetes, and aging on cognition: implications for practice and research. J Neurosci Nurs. 2014; 46(5):292-305. [PubMed: 25099061]

59. Bale TL, Epperson CN. Sex differences and stress across the lifespan. Nat Neurosci. 2015; 18(10): 1413-1420. [PubMed: 26404716]

60. Lee C, Tsenkova V, Carr D. Childhood trauma and metabolic syndrome in men and women. Soc Sci Med. 2014; 105:122-130. [PubMed: 24524907]

61. Tsenkova V, Pudrovska T, Karlamangla A. Childhood socioeconomic disadvantage and prediabetes and diabetes in later life: a study of biopsychosocial pathways. Psychosom Med. 2014; 76(8):622628. [PubMed: 25272201]

62. Cohen M, Deamant C, Barkan S, et al. Domestic violence and childhood sexual abuse in HIVinfected women and women at risk for HIV. Am J Public Health. 2000; 90(4):560-565. [PubMed: 10754970]

63. Aggarwal NT, Wilson RS, Beck TL, et al. Perceived stress and change in cognitive function among adults 65 years and older. Psychosom Med. 2014; 76(1):80-85. [PubMed: 24367123]

64. Osmanovic-Thunstrom A, Mossello E, Akerstedt T, Fratiglioni L, Wang HX. Do levels of perceived stress increase with increasing age after age 65? A population-based study. Age Ageing. 2015; 44(5):828-834. [PubMed: 26187986]

65. Valcour V, Paul R, Neuhaus J, Shikuma C. The Effects of Age and HIV on Neuropsychological Performance. J Int Neuropsychol Soc. 2011; 17(1):190-195. [PubMed: 21144102]

66. van Gorp WG, Miller EN, Marcotte TD, et al. The relationship between age and cognitive impairment in HIV-1 infection: findings from the Multicenter AIDS Cohort Study and a clinical cohort. Neurology. 1994; 44(5):929-935. [PubMed: 8190299]

67. Vance DE, Fazeli PL, Gakumo CA. The impact of neuropsychological performance on everyday functioning between older and younger adults with and without HIV. J Assoc Nurses AIDS Care. 2013; 24(2):112-125. [PubMed: 22943982]

68. Sheppard DP, Woods SP, Bondi MW, et al. Does Older Age Confer an Increased Risk of Incident Neurocognitive Disorders Among Persons Living with HIV Disease? Clin Neuropsychol. 2015; 29(5):656-677. [PubMed: 26367342]

69. Maillet D, Rajah MN. Association between prefrontal activity and volume change in prefrontal and medial temporal lobes in aging and dementia: a review. Ageing Res Rev. 2013; 12(2):479-489.

[PubMed: 23183352] 


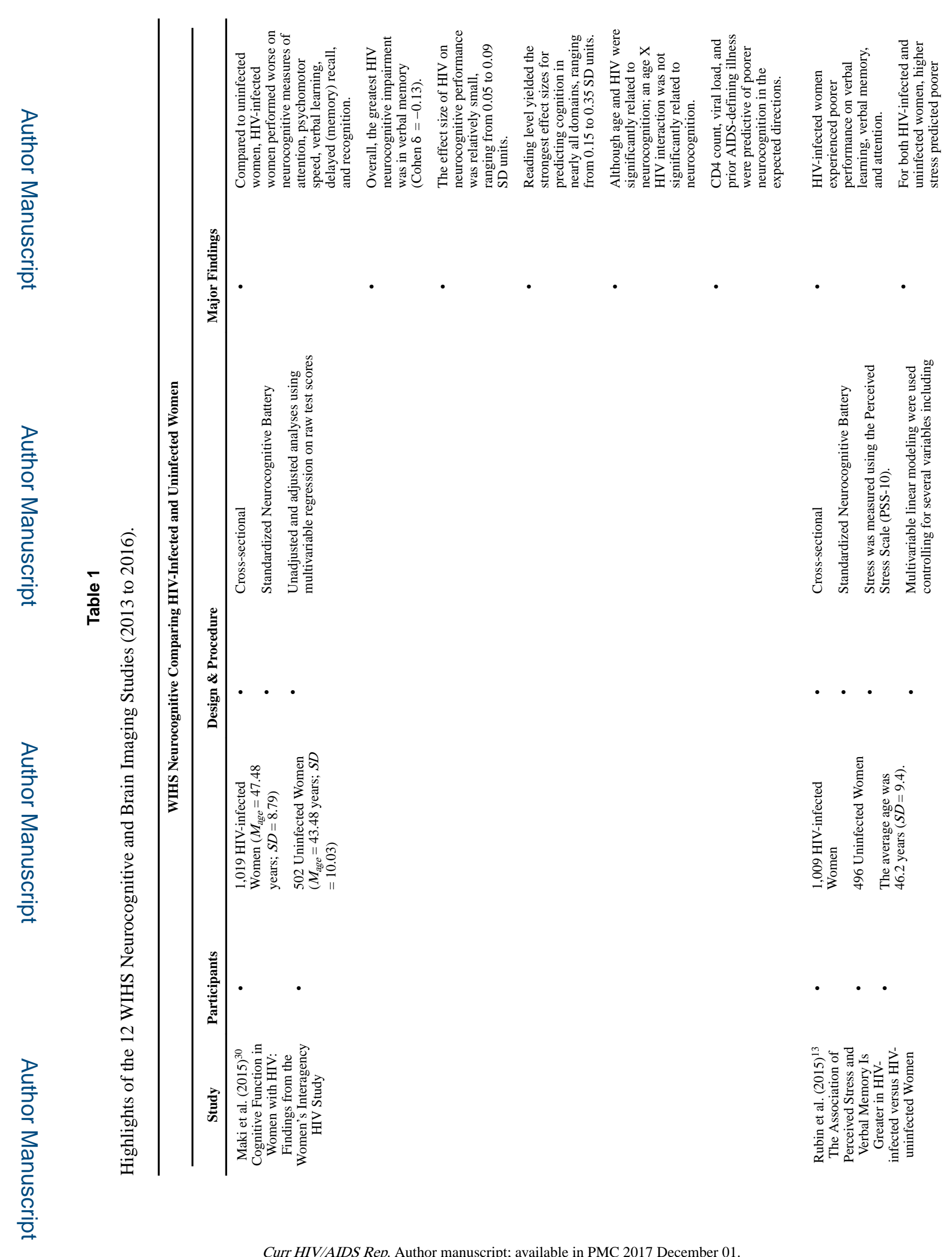




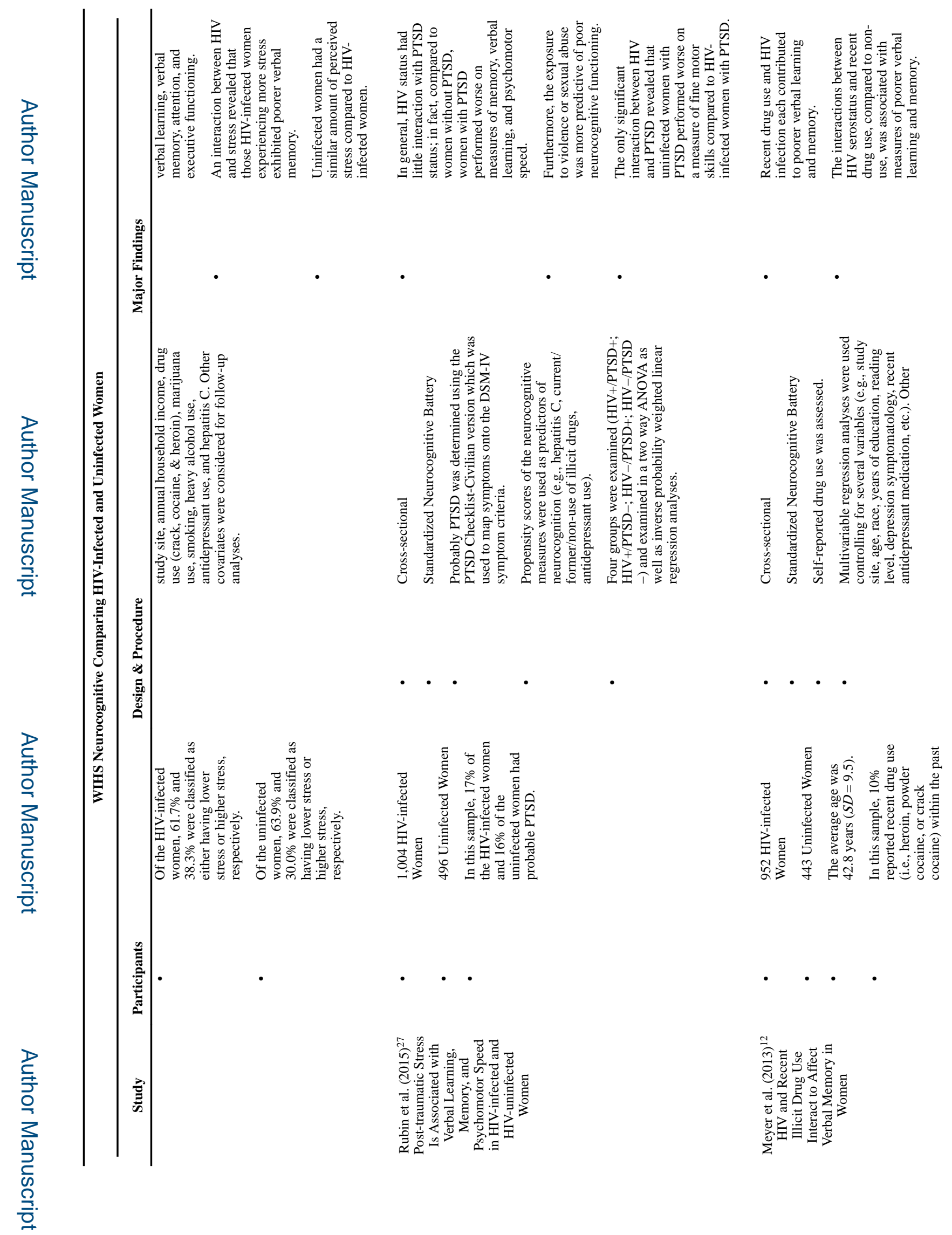

Curr HIV/AIDS Rep. Author manuscript; available in PMC 2017 December 01. 


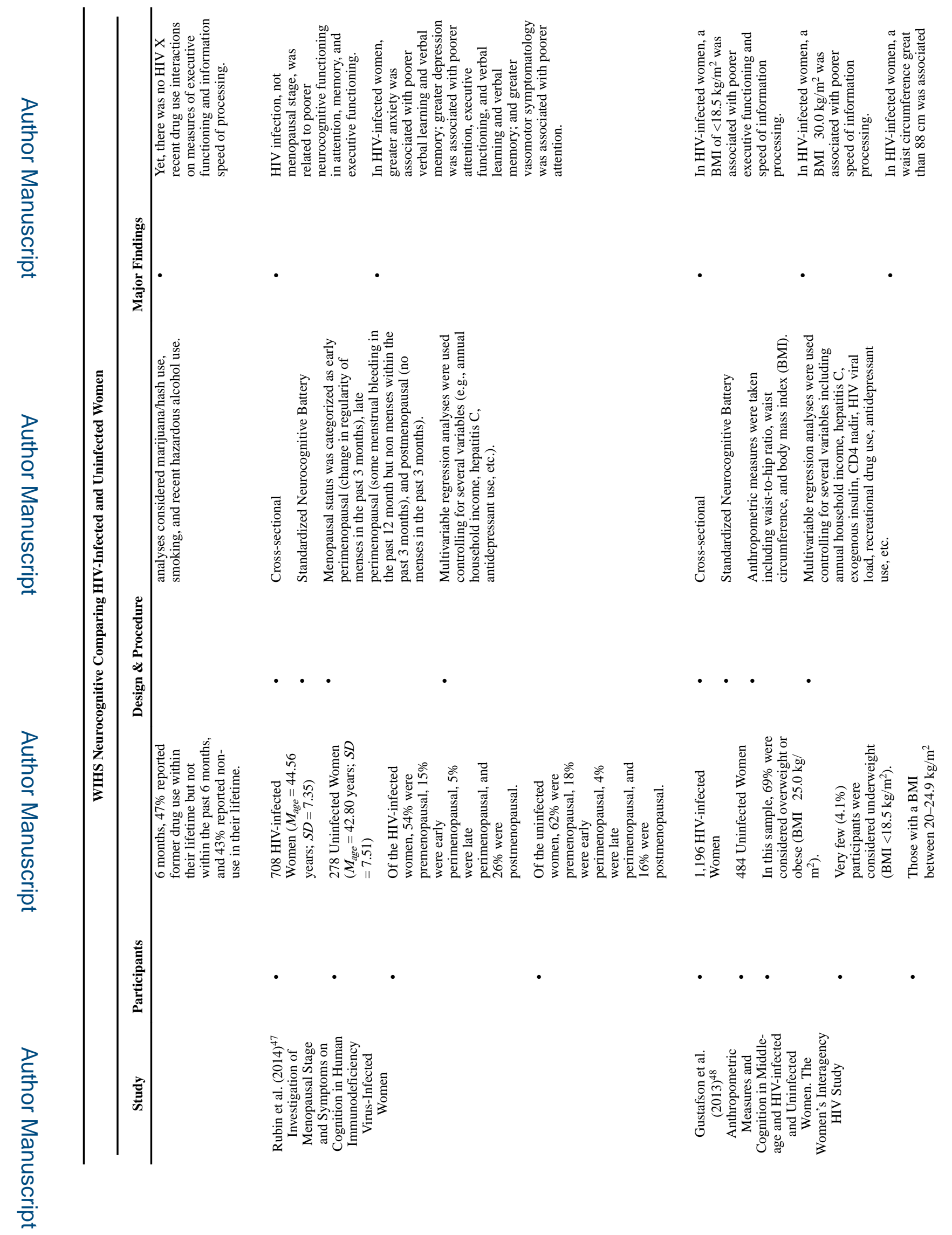

Curr HIV/AIDS Rep. Author manuscript; available in PMC 2017 December 01. 


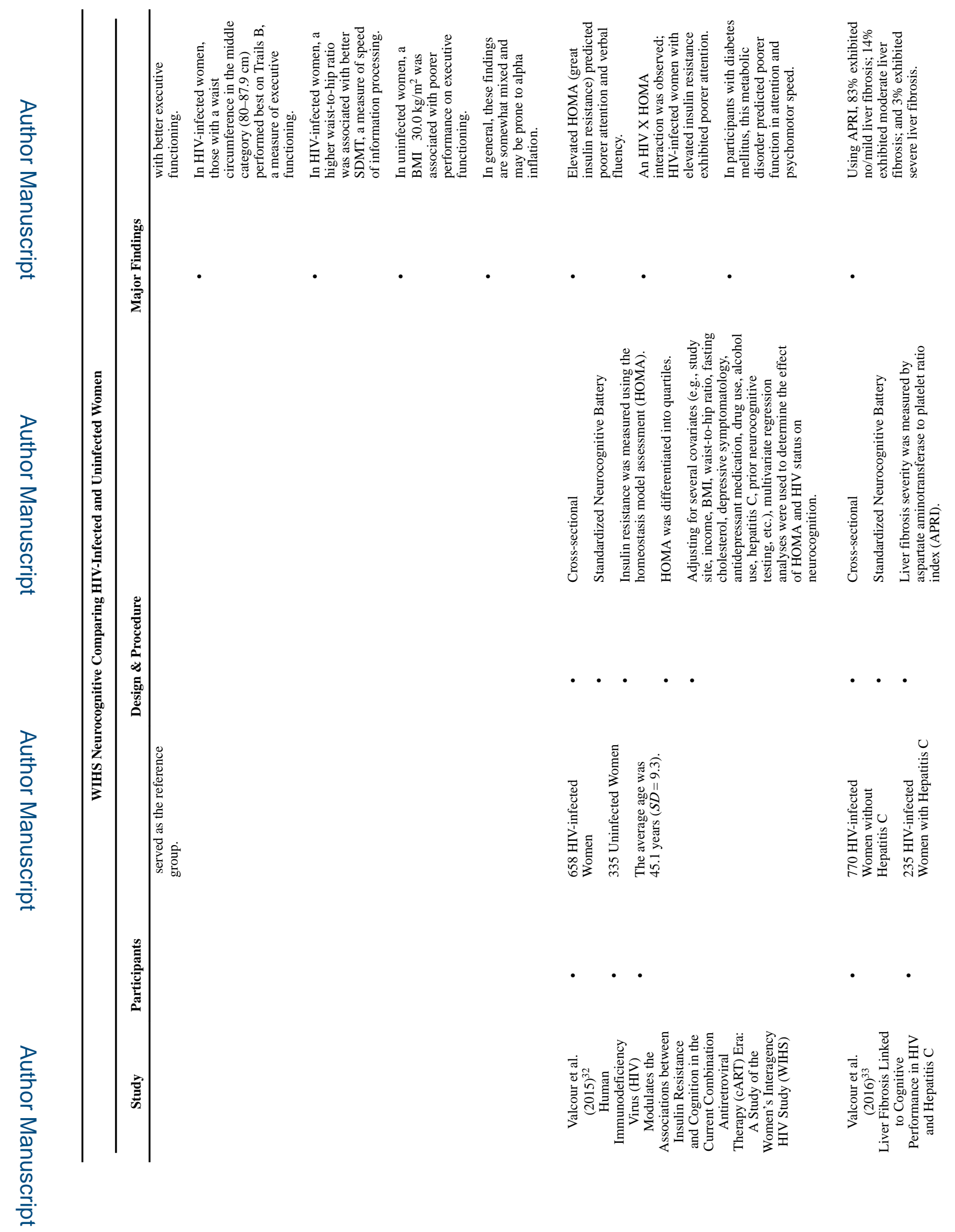

Curr HIV/AIDS Rep. Author manuscript; available in PMC 2017 December 01. 
Vance et al.

Page 20

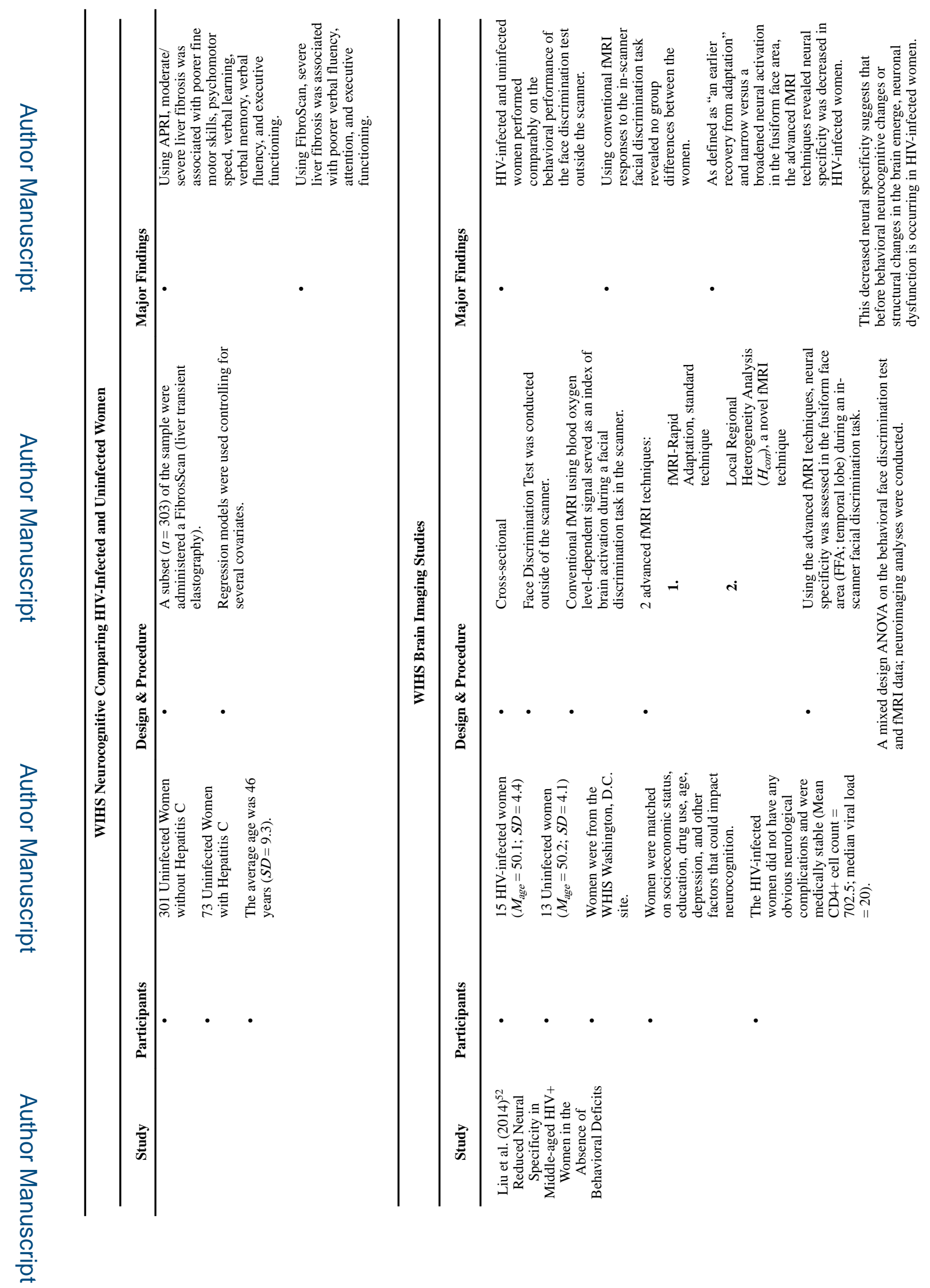

Curr HIV/AIDS Rep. Author manuscript; available in PMC 2017 December 01. 


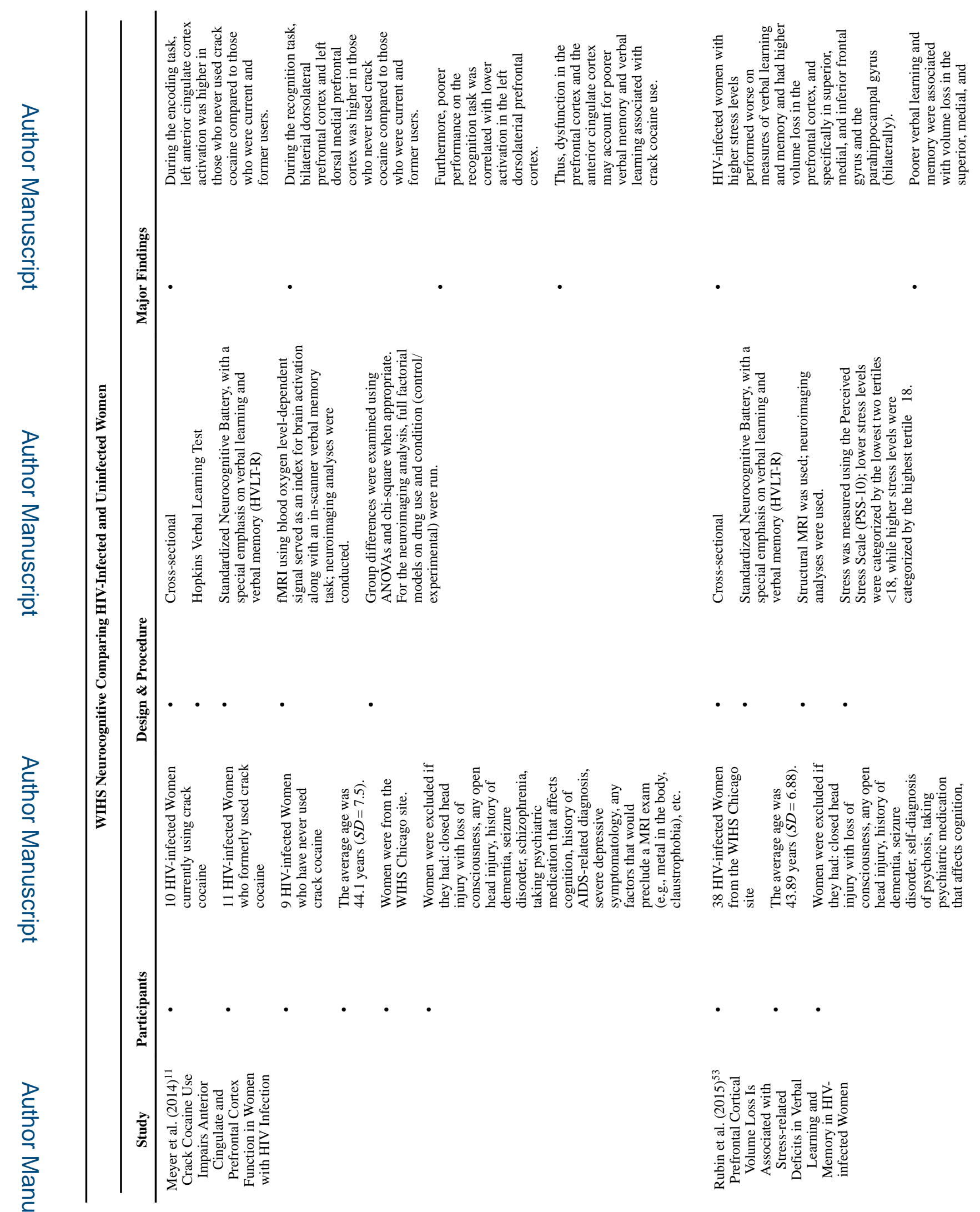


Vance et al.

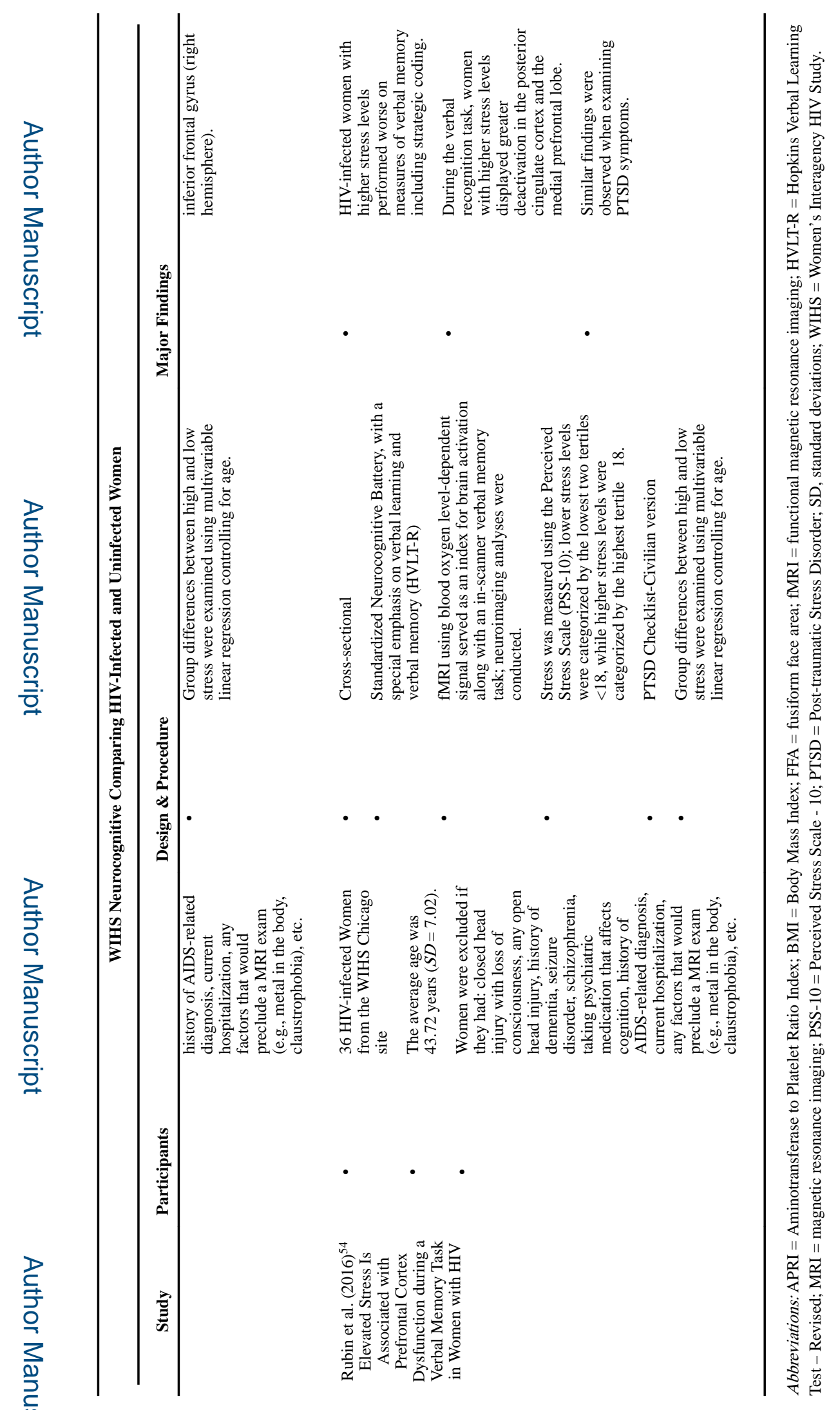

Curr HIV/AIDS Rep. Author manuscript; available in PMC 2017 December 01. 